\section{Pentagon breaks FDA rules}

On January 12th, a deadline set by the US Food and Drug Administration (FDA) for the Department of Defense (DOD) to explain why serious deviations occurred in a vaccination protocol for troops in Bosnia, expired. The DOD did not respond and had not done so by the time that this issue of Nature Medicine went to press.

The incident in question was the 1996 immunization of 3,981 military personnel against tickborne encephalitis (TBE)-a viral infection of the central nervous system which causes flu-like symptoms and can lead to complications such as swelling of the brain and coma. Although a safe and effective vaccine for TBE is produced in Europe, the non-existence of the disease in the US means that the vaccine has never been tested there. Thus, it was administered under an Investigational New Drug (IND) protocol in Bosnia. Errors in that schedule were revealed in an on-site FDA inspection in June last year.

This, and similar events observed in the medical treatment of military personnel in the Gulf War, is causing some to question the Army's ability to perform the massive immunization programs that seem increasingly likely under the specter of biological warfare.

After examining the Bosnian TBE program, the FDA listed several deviations from federal regulations in a July 1997 letter sent to Edward Martin, Acting Assistant Secretary of Defense for Health Affaires at the Pentagon. Overall, it appears that the Army medical corps failed to maintain adequate records showing the receipt, shipment and disposition of the TBE vaccine. Over ten thousand doses were sent to Bosnia, but DOD records could not account for 3,065 of these. In addition, the DOD was known to have experimented with the TBE vaccination schedule, but these tests were never monitored. Another major criticism was that the language on informed consent documents given to military personnel prior to immunization did not outline the possible risks of the vaccine-admittedly minimal in the case of TBE.

The letter, signed by FDA Lead Deputy Commissioner Michael Friedman, stated that the deviations "point to an underlying inability of DOD to carry out its oblig- ations under INDs for drugs and biologics intended to provide potential protection to deployed military personnel."

In his reply, Lieutenant General Ronald Blanck remarked that the TBE vaccine was administered for the purpose of "force protection and not to obtain safety and efficacy data." He countered that the majority of the missing 3,065 vaccine doses were, in fact, destroyed and that only 242 remain unaccounted for. But Blanck's response was deemed "insufficient" and the FDA demanded a complete written response by January 12th, 1998.

It is widely appreciated that the battlefield is not a clinical setting: troop locations are switched overnight and warfare, not medical schedules, is priority. However, airtight medical documentation is essential if the origin of any subsequent illness is to be traced. This matter becomes more pertinent when a treatment is new and/or has not been tested extensively. There is currently a range of new vaccines against potential biological agents in development. High priority $R \& D$ programs at USAMRIID include recombinant vaccines against clostridium botulinum and staphylococcus aureus endotoxins. The military will probably be the first group to receive these on a large scale in what would amount to a Phase III clinical trial.

On December 15th, the Pentagon announced that 1.4 million troops and one million national reserves will be vaccinated against anthrax over the next six years, beginning this summer, at a total cost of $\$ 130$ million. The Army insists that the anthrax vaccine has been widely tested since the early 1970 s by livestock workers and vetCOPYRI erinarians and exhibits fewer side GHT effects than flu or typhoid vaccines. REASO Moreover, the military claims to have immunized around 3,000 perNS sonnel assigned to special operations units with the vaccine.

However, Leonard Cole, adjunct professor of political science at Sheila foran Rutgers University, New Jersey and an expert on biological and chemical warfare, retains a healthy skepticism. Cole argues that despite these peace-time vaccinations, none of the recipients were immunologically challenged: "We still

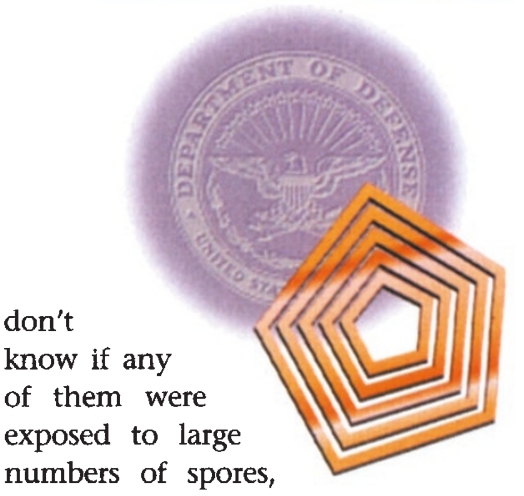

which would be the case with a biological weapon and which is the only true measure of efficacy of the vaccine."

He is worried that although 150,000 troops received the anthrax vaccine on the eve of the Gulf War, which should have contributed data on the vaccine, the inaccuracy and incompleteness of the Army's records, which has been recognized by National Institute of Medicine and Congressional inquiries, make analysis impossible. Add to this a less than simple immunization protocol for the anthrax vaccine-six inoculations over an 18-month period, followed by an annual booster for full protection-and the situation is troubling.

Cole is not against a vaccine, he supports one. But he believes that the best way to proceed is to closely monitor immunization in a few thousand military people before proceeding with mass immunization. Cole also advises oversight of future military protocols by a non-military supervisor.

On the surface, it appears that the Pentagon has learned lessons form the Gulf War. It has stated that anthrax immunization would start only after several conditions are met, including "implementation of a system for fully tracking personnel who receive the vaccinations" and a "review of health and medical issues of the program by an independent expert." Gerald N. Burrow of Yale University has been appointed to act as an independent assessor of the program.

But a paragraph in Friedman's letter, referring to DOD claims-made before the TBE vaccination incident-that it had "learned lessons" from the Gulf War, should serve as a warning. Friedman was forced to conclude that the Bosnian deviations "show that DOD has not corrected its procedures to prevent the recurrence of problems in the use of investigational products that arose during the Persian Gulf War."

KAREN BIRMINGHAM, NEW YORK 